\title{
The accuracy of medical dispatch - a systematic review
}

K. Bohm ${ }^{1,2^{*}}$ and L. Kurland ${ }^{3,4}$

\begin{abstract}
Background: It is a challenge to dispatch Emergency medical Services (EMS) appropriately with limited resources and maintaining patient safety; this requires accurate dispatching systems. The objective of the current systematic review was to examine the evidence, according to GRADE, for medical dispatching systems to accurately dispatch EMS according to level of acuity and in recognition of specific conditions.

A systematic search was performed trough PubMed, Web of Science, Embase (free text in all fields), Centre for Reviews and Dissemination (CRD), and Cochrane Central Register of Controlled Trials up to 16th of May, 2017. A combination of keywords and Medical Subject Heading (MeSH) terms relevant to "emergency medical dispatch criteria" were used, to search for articles published between 2012 and 2017. Publications were included according to the inclusion/exclusion criteria using the Systematic Reviews and Meta-Analyses (PRISMA) protocol. Level of evidence was evaluated in accordance with Grading of Recommendations Assessment, Development and Evaluation (GRADE). Articles included were those that provided evidence for at least one of the measures of dispatch system accuracy; i.e. sensitivity, specificity, positive and negative predictive and/or over- and under-triage. The search identified 1445 articles. After the removal of duplicates, 382 titles were reviewed for relevance and an additional 359 articles were excluded based on manuscript title and abstract. An additional five articles were excluded after review of the full text versions of the remaining articles. The current review included 18 publications which all were based on primary research.

Conclusions: The 18 articles addressed the identification of cardiac arrest, stroke, medical priority and major trauma using different dispatching systems. The results of the current review show that there is a very low to low overall level of evidence for the accuracy of medical dispatching systems. We suggest that it is necessary to create a consensus on common standards for reporting before consensus can be reached for the level of accuracy in medical dispatching systems.
\end{abstract}

Keywords: Emergency medical dispatch, Emergency medical services, Medical order entry systems

\section{Background}

The objective for the telecommunicator at the dispatch center is - based on the information obtained during a telephone call - to evaluate whether emergency medical services (EMS) are needed and with which priority the resource needs to be dispatched [1]. The challenge is to dispatch EMS appropriately with limited resources and still be safe for the patients; this requires accurate dispatching systems.

There are several types of dispatching systems but they can be categorized as two types of systems; the Medical

\footnotetext{
* Correspondence: katarina.bohm@ki.se

${ }^{1}$ Department of Clinical Science and Education, Södersjukhuset, Karolinska Institutet, SE 11883 Stockholm, Sweden

2Department of Emergency Medicine, Södersjukhuset, Stockholm, Sweden

Full list of author information is available at the end of the article
}

Priority Dispatch system (MPDS) [2, 3] mainly used in Anglo-Saxon countries, and the criteria-based dispatch (CBD) $[4,5]$ used in Nordic and European countries. Common for both systems is that the telecommunicator allocates each call to one of the listed chief complaints. While MPDS is based on codes and scripted questions to put to the caller, the CBD system relies on the experience of the telecommunicator to conduct the interview. In addition to the different systems for medical dispatching, there are also different systems for the EMS response. The EMS organization can have e.g. advanced and/or basic life support ambulances, first responders or pre-hospital emergency physicians and helicopter emergency services (HEMS). However, the accuracy of EMS systems, which

(c) The Author(s). 2018 Open Access This article is distributed under the terms of the Creative Commons Attribution 4.0 International License (http://creativecommons.org/licenses/by/4.0/), which permits unrestricted use, distribution, and reproduction in any medium, provided you give appropriate credit to the original author(s) and the source, provide a link to the Creative Commons license, and indicate if changes were made. The Creative Commons Public Domain Dedication waiver (http://creativecommons.org/publicdomain/zero/1.0/) applies to the data made available in this article, unless otherwise stated. 
per definition includes both dispatching and the response to dispatching are not systematically described.

Dispatching accuracy, or effectiveness, relates to the ability of the dispatching system to discriminate between the required EMS resources and the priority of these. Measures of accuracy are both discriminative, e.g. sensitivity and specificity, and predictive, e.g. positive predictive value and negative predictive value [6]. Other relevant measures of performance of dispatching systems are over- and under-triage [7]. While our systems are geared towards over-triage so as not to miss critical patients in need of medical interventions, i.e. to avoid under-triage, over-triage consumes resources and increases the risk for occupational injuries of health care personnel. There is, however, no consensus on levels for over-and under-triage or dispatching accuracy.

The objective of the current systematic review was to examine the evidence, according to GRADE, for medical dispatching systems to accurately dispatch EMS according to level of acuity and in recognition of specific conditions. Accuracy was measured as sensitivity, specificity, positive and negative predictive value in addition to over- and under-triage.

\section{Methods}

\section{Search strategy}

The current systematic review includes the identification of articles according to Preferred Reporting Items for Systematic Reviews and Meta-Analyses (PRISMA) criteria [8]. The identification of publications included in the current review was made through a systematic search of the PubMed, Web of Science, Embase (free text in all fields), Centre for Reviews and Dissemination (CRD), and Cochrane Central Register of Controlled Trials up to 16th of May, 2017. A combination of keywords and Medical Subject Heading (MeSH) terms relevant to "emergency medical dispatch criteria", published in the last 5 years, was used with the assistance of a librarian (Table 1).

\section{Inclusion- and exclusion criteria}

Publications were included in the systematic review if they presented primary data which evaluated the accuracyof medical dispatch systems in current use and provided evidence for at least one of the measures of dispatch system accuracy; i.e. sensitivity, specificity, positive predictive value (PPV) and negative predictive value (NPV) and/or over- and under-triage. Publications evaluating dispatch/ triage for military resources, mass casualty/disaster and inter-facility transfers were excluded. The search was limited to studies on humans, published in English.
Table 1 Search string.

\begin{tabular}{cl}
\hline PubMed & \\
1 & (medical[all fields] AND dispatch*[all fields]) \\
& OR (emergency[all fields] AND dispatch*[all fields]) \\
& OR "Emergency Medical Dispatch"[all fields] \\
& OR dispatch centres[all fields] \\
2 & triage[all fields] \\
3 & Criteria based[all fields] \\
4 & Physician based[all fields] \\
5 & "emergency medicine" [all fields] \\
6 & 2 OR 3 OR 4 OR 5 \\
7 & 1 AND 6 \\
8 & English, year> = 2012
\end{tabular}

Web of Science (Indexes = SCI-EXPANDED, SSCI,

A\&HCl, ESCl Timespan = All years)

\begin{tabular}{|c|c|}
\hline 1 & Topic $=$ (Emergency OR Medical OR centres) AND Dispatch* \\
\hline 2 & Topic $=$ triage \\
\hline 3 & Topic $=$ criteria based \\
\hline 4 & Topic $=$ physician based \\
\hline 5 & Topic "emergency medicine" \\
\hline 6 & 2 OR 3 OR 4 OR 5 \\
\hline 7 & 1 AND 6 \\
\hline 8 & 7 AND English, year > = 2012, Article, Review \\
\hline \multicolumn{2}{|c|}{ mbase (free text in all fields) } \\
\hline 1 & (emergency OR medical OR centres) AND dispatch* \\
\hline 2 & Triage \\
\hline 3 & criteria based \\
\hline 4 & physician based \\
\hline 5 & "emergency medicine" \\
\hline 6 & 2 OR 3 OR 4 OR 5 \\
\hline 7 & 1 AND 6 \\
\hline 8 & 7 AND English, year $>=2012$, article, review, article in press \\
\hline \multicolumn{2}{|c|}{ RD - Centre for Reviews and Dissemination, York } \\
\hline 1 & Dispatch*, english, year > = 2012 \\
\hline \multicolumn{2}{|c|}{ ochrane } \\
\hline 1 & (emergency OR medical OR centres) AND dispatch* \\
\hline 2 & Triage OR criteria OR physician OR "emergency medicine" \\
\hline 3 & 1 AND 2 \\
\hline 4 & 3 AND english, year $>=2012$, NOT conference \\
\hline
\end{tabular}

\section{Title and abstract screening}

The titles and abstracts were screened independently by the two authors. Inclusion in the subsequent full-text review was made through discussion and consensus.

\section{Full text screening for relevance}

The selected full-text articles were reviewed independently on the basis of inclusion and exclusion criteria. Relevant articles were reviewed to determine whether 
they provided evidence for at least one of the measures of dispatch system accuracy; i.e. sensitivity, specificity, positive predictive value (PPV) and negative predictive value (NPV) and/or over- and under-triage.

\section{Level of evidence according to GRADE}

Publications were reviewed in detail and the overall quality of evidence was based on the recommendations of the Grading of Recommendations Assessment, Development and Evaluation (GRADE) working group [9]. The level of evidence was categorized as 'very low, 'low', 'moderate, 'high' or 'very high' in accordance with GRADE [10] with special emphasis on diagnostic tests [10]. Briefly; retrospective studies are graded as very low or low, while high or very high require a prospective study design [10]. Factors that determine and can decrease the quality of evidence are study design, risk of bias, indirectness, inconsistency in study results, imprecise evidence and publication bias [10].

\section{Measurement of inter-rater agreement}

The kappa coefficient was calculated to study the agreement between the observers ability to classify titles and abstracts (yes/no) [11].

\section{Results}

\section{Study selection}

The search identified 1445 publications. After the removal of duplicates, 382 titles were reviewed for relevance and an additional 359 citations were excluded based on manuscript title and abstract. An additional five articles were excluded (three non-dispatch studies and two with no primary data) after review of full text of the remaining publications. The current review included 18 publications. The PRISMA flow diagram summarizes the inclusion/exclusion process, Fig. 1.

\section{Inter-rater agreement}

The $\mathrm{k}$ values, were 0.53 (95\% CI; 0.45-0.62) for comparison of titles and 0.68 (95\% CI; 0.50-0.86) for comparison of abstracts. The latter is considered as 'substantial' agreement between the raters [12].

\section{Characteristics of included articles}

Characteristics of the 18 included publications are presented in Table 2. All included publications were primary research. It was not possible to perform a meta-analysis due to the heterogeneity of the studies included in the current systematic review. The results are presented below in relation to their study populations and main objectives, i.e. identification of cardiac arrest, stroke, medical priority and helicopter medical services dispatching for major trauma.

The main results; the accuracy for dispatching systems is presented in Table 3, along with the results for overand under-triage. Three articles presented all measures [13-15].

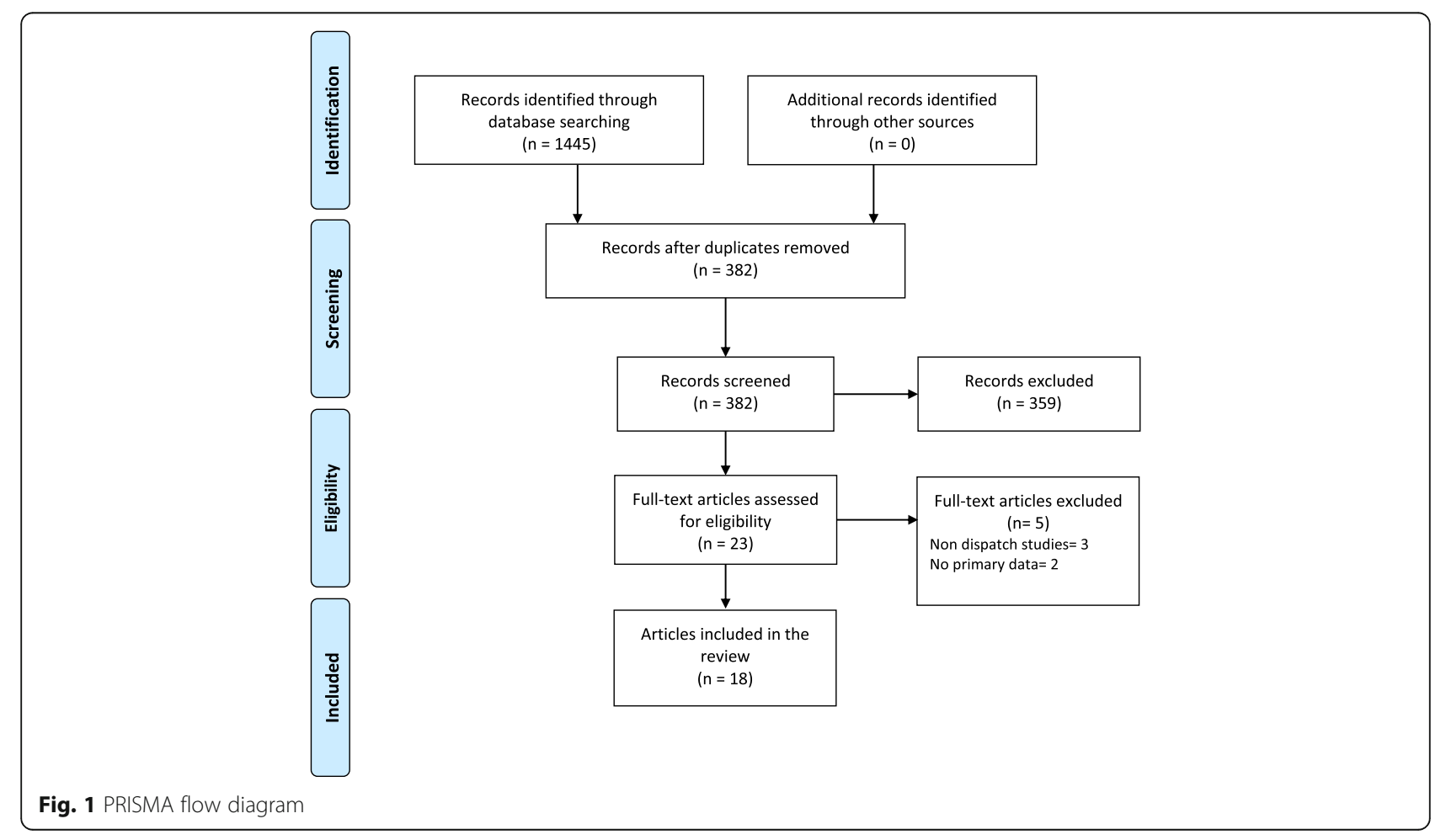




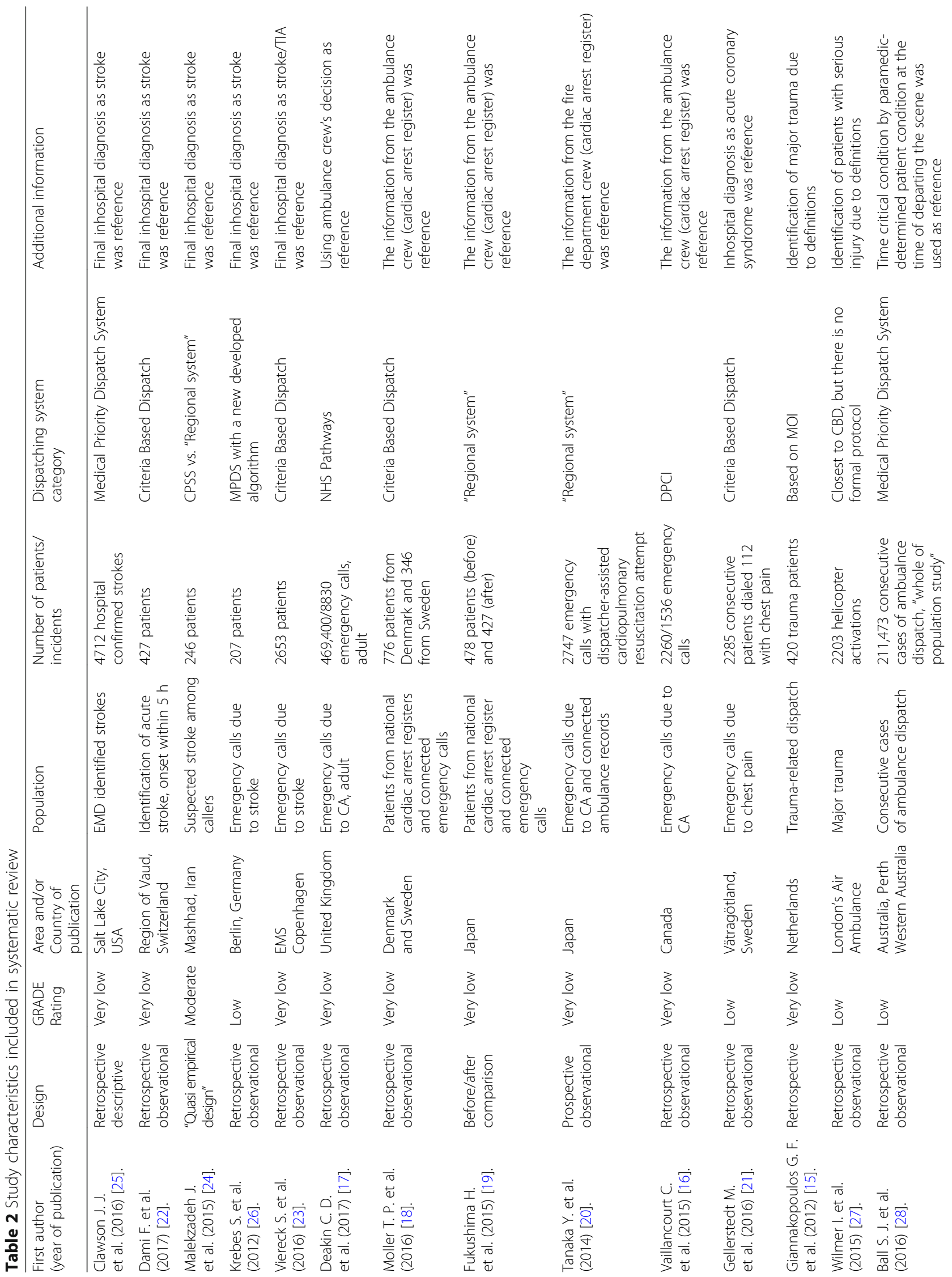




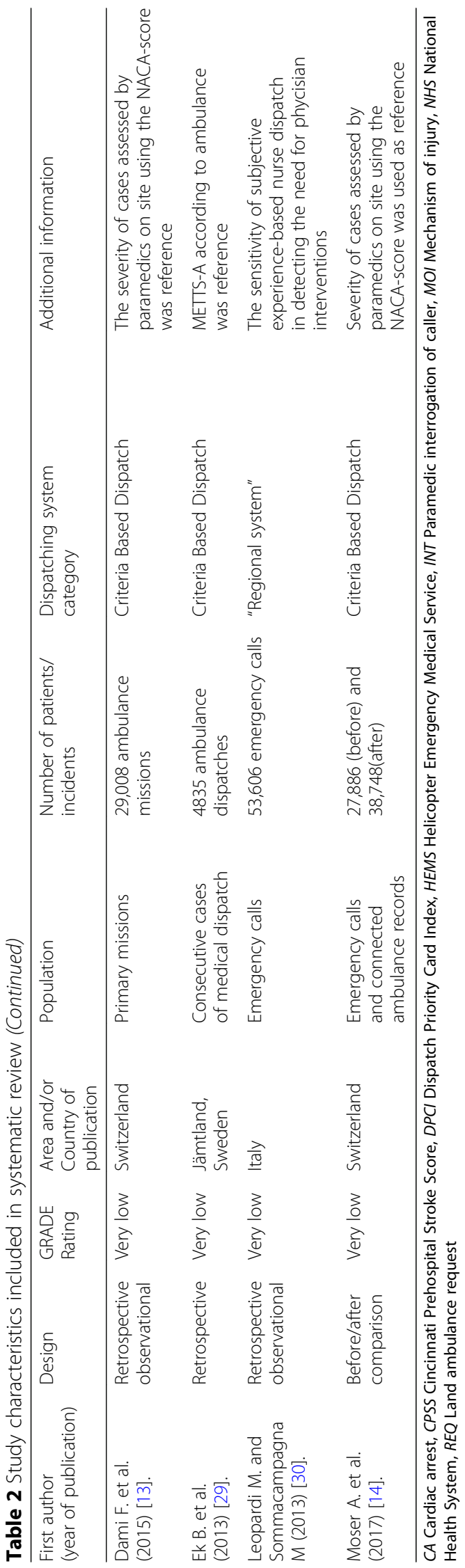


Table 3 Measures for dispatch accuracy per included study

\begin{tabular}{|c|c|c|c|c|c|c|c|}
\hline Category & Author, year & Sensitivity \% (95\% Cl) & $\begin{array}{l}\text { Specificity } \\
\%(95 \% \text { Cl) }\end{array}$ & $\begin{array}{l}\text { PPV \% } \\
(95 \% \text { Cl) }\end{array}$ & $\begin{array}{l}\text { NPV \% } \\
(95 \% \text { Cl) }\end{array}$ & Over-triage \% (95\% Cl) & $\begin{array}{l}\text { Under-triage } \\
\%(95 \% \mathrm{Cl})\end{array}$ \\
\hline \multirow[t]{5}{*}{ Stroke } & $\begin{array}{l}\text { Clawson } \\
\text { et al., } 2016\end{array}$ & 86.4 & 26.6 & 20.0 & 90.2 & & \\
\hline & $\begin{array}{l}\text { Dami } \\
\text { et al., } 2017\end{array}$ & $67.8(54.3-79.4)$ & $\begin{array}{l}98.6 \\
(98.4-98.7)\end{array}$ & $9.4(6.6-12$. & $\begin{array}{l}99.9 \\
(99.9-99.9)\end{array}$ & & \\
\hline & $\begin{array}{l}\text { Malekzadeh } \\
\text { et al., } 2015\end{array}$ & & & & & 11.6 vs 20.8 & 10.7 vs 13.6 \\
\hline & $\begin{array}{l}\text { Krebes } \\
\text { et al., } 2012\end{array}$ & $\begin{array}{l}\text { Stroke: } 53.3 \\
(47.0-59.0)\end{array}$ & 97 (97-98) & $\begin{array}{l}\text { Stroke: } 47.8 \\
(42.0-54.0)\end{array}$ & $\begin{array}{l}98 \\
(97-98)\end{array}$ & & \\
\hline & $\begin{array}{l}\text { Viereck } \\
\text { et al., } 2016\end{array}$ & $66.2(64.4-68.0)$ & & $\begin{array}{l}30.2 \\
(29.1-31.4)\end{array}$ & & & \\
\hline \multirow[t]{5}{*}{ Cardiac arrest } & $\begin{array}{l}\text { Deakin } \\
\text { et al., } 2017\end{array}$ & $75.9(74.3-77.3)$ & $\begin{array}{l}98.6 \\
(98.6-98.7)\end{array}$ & $\begin{array}{l}26.8 \\
(25.88-27.7)\end{array}$ & $\begin{array}{l}99.8 \\
(99.82-99.85)\end{array}$ & & \\
\hline & $\begin{array}{l}\text { Möller } \\
\text { et al., } 2016\end{array}$ & $\begin{array}{l}\text { Copenhagen } 80.7 \\
\text { (77.7-84.3), } \\
\text { Skåne } 86.0 \text { (81.3-89.8) }\end{array}$ & & & & & \\
\hline & $\begin{array}{l}\text { Fukushima } \\
\text { et al., } 2015\end{array}$ & 93 & 50 & & & & \\
\hline & $\begin{array}{l}\text { Tanaka } \\
\text { et al., } 2014\end{array}$ & $72.9(71.7-74.1)$ & $\begin{array}{l}99.6 \\
(99.6-99.6)\end{array}$ & & & & \\
\hline & $\begin{array}{l}\text { Vaillancourt } \\
\text { et al., } 2015\end{array}$ & $65.9(63.5-68.2)$ & $\begin{array}{l}32.3 \\
(29.0-35.9)\end{array}$ & 67.4 & 30.9 & & \\
\hline $\begin{array}{l}\text { Acute coronary } \\
\text { syndrome }\end{array}$ & $\begin{array}{l}\text { Gellerstedt } \\
\text { et al., } 2016\end{array}$ & 82.6 & & & & & \\
\hline \multirow[t]{4}{*}{ Major trauma } & $\begin{array}{l}\text { Giannokopoulos } \\
\text { et al., } 2012\end{array}$ & 87.7 & 45.3 & 48.4 & 86.3 & 44 & 20.6 \\
\hline & \multirow{3}{*}{$\begin{array}{l}\text { Wilmer } \\
\text { et al., } 2015\end{array}$} & \multirow[t]{3}{*}{ MOI + INT: 80.2} & & & & MOl: 41.2 & REQ/all: 19.7 \\
\hline & & & & & & INT: 30.2 & \\
\hline & & & & & & REQ: 27.7 & \\
\hline \multirow[t]{5}{*}{ Medical priority } & $\begin{array}{l}\text { Ball } \\
\text { et al., } 2016\end{array}$ & 93.32 (92.71-93.89) & $\begin{array}{l}48.6 \\
(48.45-48.89)\end{array}$ & & & & \\
\hline & $\begin{array}{l}\text { Dami } \\
\text { et al., } 2015\end{array}$ & $86(85.6-86.4)$ & 48 (47.4-48.6) & $\begin{array}{l}21.7 \\
(21.2-22-2)\end{array}$ & $\begin{array}{l}95.4 \\
(95.2-95.6)\end{array}$ & 78 & 4.6 \\
\hline & $\begin{array}{l}\text { Ek } \\
\text { et al., } 2013\end{array}$ & 93.32 & 15.4 & & & & \\
\hline & $\begin{array}{l}\text { Leopardi } \\
\text { et al., } 2013\end{array}$ & 78.0 (76.9-79.1) & $\begin{array}{l}83.8 \\
(83.4-84.1)\end{array}$ & $\begin{array}{l}36.6 \\
(35.8-37.5)\end{array}$ & $\begin{array}{l}96.9 \\
(96.8-97.1)\end{array}$ & & \\
\hline & $\begin{array}{l}\text { Moser } \\
\text { et al., } 2017\end{array}$ & $A+B 86.8(86.5-87.1)$ & $\begin{array}{l}A+B \text { 67.4 } \\
(66.9-67.9)\end{array}$ & $\begin{array}{l}29.2 \\
(28.7-29.7)\end{array}$ & $\begin{array}{l}97.0 \\
(70.3-71.3)\end{array}$ & $\begin{array}{l}\text { After } 70.8 \\
\text { (70.3-71.3) }\end{array}$ & $\begin{array}{l}\text { After } 3.0 \\
(2.8-3.2)\end{array}$ \\
\hline
\end{tabular}

CI Confidence Interval, DPCI Dispatch Priority Card Index, HEMS Helicopter Emergency Medical Service, INT Paramedic interrogation of caller, MPDS Medical Priority Dispatch System, MOI Mechanism of injury, NHS National Health System, NPV Negative Predicted Value, PPV Positive Predicted Value, REQ Land ambulance request

\section{Identification of cardiac arrest}

The overall sensitivity for identifying cardiac arrest was 65.9\% [16], 75.9\% [17], 80.7 and $86.0 \%$ at two different sites [18], respectively. In two studies, the sensitivity was 93.0\% [19] and 72.9\% [20] after implementation of modified protocols. These five organizations used five different systems/protocols; NHS Pathways [Deakin], Criteria Based Dispatch (CBD) [20] and Dispatch Priority Card Index (DPCI) [16], and two different Japanese protocols [19, 20]. The corresponding specificity was 32.3\% [16], 50.0\% [19], 98.6\% [17], and 99.6\% [20] respectively. The PPV was reported in one of the cardiac arrest-studies as $26.8 \%$ (95\% CI 25.9-27.7\%) [17]. In these five studies the sensitivity and specificity relate to identification of cardiac arrest among patients that the ambulance personnel reported as cardiac arrest, not to a sample of unselected calls.

\section{Identification of acute coronary syndrome (ACS)}

Among patients calling with chest pain, Gellerstedt et al. demonstrated a sensitivity of 82.6 and $17.4 \%$ false negatives when identifying acute coronary syndrome [21]. 


\section{Identification of stroke}

The sensitivity for identifying stroke was $67.8 \%$ [22], $66.2 \%$ [23] and 77.7\% [24], all three articles using local adaptations of the Cincinnati Prehospital Stroke Score, and $86.4 \%$ [25] using the MPDS Stroke Diagnostic Tool. While the specificity was 26.6\% [25] for the Stroke Diagnostic Tool. Krebes et al. implemented a new algorithm based on the MPDS algorithm, and reported a sensitivity of $53.3 \%$ [26].

The PPV was $20.0 \%$ and the NPV $90.2 \%$ for the Stroke Diagnostic Tool [25], and 30.2\% PPV for the adapted Cincinnati Prehospital Stroke Score [23]. The PPV was $47.8 \%$ with the new algorithm by Krebes [26].

Over-triage was $11.6 \%$ for the adapted Cincinnati Prehospital Stroke Score and $20.8 \%$ for the National Guidelines for Telephone Triage Tool and under-triage 10.7 and $13.6 \%$ respectively [24].

In these five articles the sensitivity and specificity relate to identification of stroke among patients with the hospital diagnosis of stroke, not to a general population of unselected calls.

\section{Identification of major trauma}

Only publications addressing the use of HEMS in the context of major trauma met with the inclusion criteria, which is why other publications addressing major trauma were not included in the current review. The dispatch criteria for HEMS had a sensitivity $87.7 \%$, a specificity of $45.3 \%$, a PPV of $48.4 \%$, and a NPV of $86.3 \%$ for the HEMS dispatch criteria to identify major trauma patients [15]. Wilmer et al. described the different dispatching methods within the same dispatching system to study the accuracy of the systems for dispatching HEMS for major trauma [27]. Mechanism of injury together with the paramedic interrogation had a sensitivity of $80.2 \%$ and under-triage of $19.7 \%$.

Two studies $[15,21]$ are in part derivation studies; i.e. studies with the aim of deriving a more accurate dispatching system. The data included in the current review from these articles is that reflecting the dispatching system in use, not the derived and unevaluated new dispatching system.

\section{Identification according to medical priority}

The overall sensitivity of identifying time critical conditions defined as ambulance dispatch priority 1 was 93.32\% [28], for dispatching priority 1 and 2 in accordance with the standard of Medical Emergency Triage and Treatment System-A, METTS-A, red, orange and yellow, 95.9\% [29]. In two studies, using Advisory Committee for Aeronautics (NACA) score, the overall sensitivity was $87 \%$ [14], and $86 \%$ [12] respectively. The sensitivity was $78.0 \%$ [30] using local criteria. While the specificity was $48 \%$ [13], 48.67\% [29], 67\% [14], 83.8\%, and [30]. Ek et al. showed a specificity of $15.4 \%$ for priority 3 dispatching in accordance with METTS-A green and blue [29].

The reported predicted values were; PPV of $36.6 \%$ (CI 35.8-37.5\%) and NPV of 96.9\% (95\% CI 96.8-97.1\%) [30]. In Dami et al. PPV was 21.7\% (21.2-22.2\%) and NPV was 95.4 (95.2-95.6\%) [12]. Ball et al. reported PPV of $5.85 \%$ (CI 5.71-5.99\%) and NPV of $0.47 \%$ (95\% CI $0.43-0.51 \%$ ) [28]. Over-triage rate was $78 \%$ [13] and $71 \%$ [14] and under-triage rate was $4.6 \%$ [13] and 3\% [14] respectively.

\section{Discussion}

The results of the current study show that there is a very low to low overall level of evidence for the accuracy of medical dispatching systems. Although all the articles included in the current systematic review are primary research, it was not possible to perform a meta-analysis due to the heterogeneity of the sample. Moreover, it is striking that only two of the 18 articles included in the current review presents information on all measures of dispatching accuracy together with over-and under-triage, and there was only one prospective study [24]. We suggest that it may be necessary to create a consensus on common standards for reporting before consensus can be formed for the level of accuracy in medical dispatching systems.

\section{Identification of cardiac arrest}

Identification of cardiac arrest is based on the recognition that the patient is unconscious and has abnormal or no breathing. Two studies in the review reported higher sensitivity following the implementation of new protocols $[19,20]$. Interestingly, both these new protocols included keywords that reflect cardiac arrest in the call between the caller and the telecommunicator. Other more novel approaches are to focus on the communication in the emergency call [31]. The observation that it is important to evaluate the communication even when measuring accuracy, was demonstrated in the study by Möller et al., the sensitivity of identifying cardiac arrest was increased by listening to the actual calls [18].

\section{Identification of stroke}

Identification of stroke has several challenges and as a result about half of the patients with stroke are identified by the medical dispatcher [32, 33]. Firstly the symptoms are often non-specific, as demonstrated by Clawson et al., in that more than one in ten patients have the chief complaint "sick person" and an additional one in ten have fallen [25]. Secondly, the objective for medical dispatching is not absolute. What is more important? Whether it is to identify an acute stroke [23-26], to identify that a patient needs to be directed to a stroke center or to identify the patient that is benefited by 
specific treatment, e.g. thrombolysis [22] or thrombectomy, remains undetermined. It is not possible to suggest a dispatching system which is superior based on the results of the current review since the level of evidence is very low and the outcome measures are different for the five included articles focusing on stroke identification.

\section{Identification of major trauma}

Only publications addressing the use of HEMS in the context of major trauma met with the inclusion criteria, which is why other publications addressing major trauma were not included in the current review. HEMS is part of the chain of care for major trauma in resource strong settings. There is evidence that HEMS is of value for multitrauma patients and patients with traumatic brain injury [34-37] and is dispatched when medical intervention is thought to be needed [15]. However, over-triage is a problem. Up to every other deployment is cancelled, predominantly by ground EMS [15]. The accuracy of the medical dispatching could be increased by including vital signs and anatomical location of injury to the mechanism of injury which is the basis for the routine HEMS dispatching system [15]. While Wilmer et al. could show that the accuracy of HEMS dispatching was superior and comparable for paramedical interrogation of caller and the assessment of need by land ambulance crew as compared to the dispatching by mechanism of injury [38]. The results of these studies lead us to believe that mechanism of injury are insufficient criteria for HEMS dispatching for major trauma, although these results need to be interpreted with caution since the level of evidence is (very) low.

\section{Identification according to medical priority}

Patients present to the telecommunicator with a wide range of symptoms, and the ultimate question is how to identify what resources are best needed for the given caller/ patient. Ball et al. considered the effect of the chief complaint in relation to over-and under-triage. The results showed that while some of the most common chief complaints are under-triaged, e.g. convulsions/ seizures and breathing problems, others are over-triaged e.g. chest pain, heart problems/ automatic defibrillator, collapse and headache. While systems with a large proportion of non-specific presentations will not be able to evaluate the system in detail [13]. Although more than half of the calls are dispatched as priority 1 - only approximately $5 \%$ of these calls are critical [27], demonstrating the large over-triage in systems, and at the same time, revealing the lack of consensus on what level over-triage level is reasonable.

There is scant evidence concerning the necessary skills and competence for the telecommunicator. An exception is the study by Leopardi et al., demonstrating that experienced nurses could assess the patients' need for advanced care as well as a medical doctor [30]. The required level of competence of telcommunicators is an area in need of further research.

\section{Over-and under-triage and the accuracy of medical dispatching systems}

We gear our emergency response systems so as not to miss patients in need of medical intervention -i.e. to avoid under-triage - and compensate by creating over-triage, i.e. "unnecessary" dispatching. Dispatching systems are e.g. "front loaded", i.e. over-triage is used as a safety rule and we assume that by creating over-triage we are "safe". However, that this is not the case is illustrated by HEMS having an over-triage of $44 \%$ and simultaneously, in the same dispatching system, an under-triage of $20 \%$ [15]. It is therefore clear that although we need to understand and set cut-off levels for over-triage (so as to avoid waste of resources and risk for personnel) and under-triage (so as to avoid potentially lifesaving interventions not being given), they are - as measures of a dispatching system - insufficient on their own.

Measures of accuracy for dispatching systems are needed as a step in the direction of getting the right treatment to the right patient at the right time. However, there is an inherent challenge to identify the subset of patients that benefit from a specific intervention e.g. HEMS or acute coronary syndrome [21,31]. In addition to making sure that e.g. the patient with a stroke can arrive in a timely fashion to the stroke center, this will also allow for telephonic support for interventions e.g. stopping a major bleeding or to perform CPR. Such studies are designed with the aim of including parameters that increase the accuracy of identification of specific conditions or diagnoses.

In addition to identifying specific conditions, it is also important to identify time critical conditions among patients presenting with a broad range of symptom presentations and to dispatch according to medical priority, i.e. without a definite diagnosis. There are no obvious answers to the best way forward. However, to agree on how to measure and report on dispatching systems is necessary in order to be able to compare different systems between different populations and settings. There are suggestions [38, 39], but these consensus documents have not been applied in the current literature, and it is time to take this a step further.

\section{Limitations}

In 2011 Fevang et al. published a consensus report on the top five research priorities in pre-hospital care [40]. Among suggested topics was dispatch system accuracy. The choice of this was based on dispatching accuracy being a well-defined aim, with defined outcome measures, pertinent operational ramifications, and an area where there was a sufficient number of published articles 
which made the systematic review possible. It is possible that the search was additionally limited by using specific search terms, however, the search was broad as presented in Table 1.

The definitions of the measures of accuracy and over-and under-triage are not the same in the included articles, which limits comparisons of the results from the different articles. Although sensitivity was defined as the probability of the medical dispatching system identifying a specific condition given that this condition is present; specificity and the predictive values did not have the same definition. Specificity was often defined in relation to a specific condition, and not in relation to an unselected sample of callers without this specific condition. Also, the definition of over- and under-triage differed between the articles. That the definition of the measures varies makes comparisons of the results difficult.

Additional factors making comparisons between the different studies difficult are e.g. that there are two in principal different categories of dispatching systems/protocols; i.e. the MPDS and the CBDS. Moreover, the responding EMS has different tiers and organizations, again; leading to a lack with respect to a golden standard for outcome measures.

The level of evidence was categorized in accordance with GRADE, and in accordance with GRADE, retrospective studies are in general very low level of evidence. Although the overall level of evidence in the articles included in the current review was very low to low; the studies are informative and often necessary in order to design future studies. Following standards e.g. those set by STARD [41] should increase the quality of evidence.

It is imperative that the data collected from the electronic health care records is both valid and reliable before we can use this data in the design of clinical decision systems for medical dispatching. None of the reviewed studies analyzed the quality of data from the health records.

\section{Conclusions}

There were 18 articles addressing the identification of cardiac arrest, stroke, medical priority and major trauma using different dispatching systems. The results of the current study show that there is an overall very low to low level of evidence for the accuracy of medical dispatching systems. We suggest that it is necessary to create a consensus on common standards for reporting before consensus can be reached for the level of accuracy in medical dispatching systems.

\section{Abbreviations}

CBD: Criteria-Based Dispatch; EMS: Emergency medical services; GRADE: Grading of Recommendations Assessment, Development and Evaluation; HEMS: Helicopter emergency services; MeSH: Medical Subject Heading; METTS-A: Emergency Triage and Treatment System-A; MPDS: Medical Priority Dispatch system; NACA: Advisory Committee for Aeronautics; NPV: Negative predictive value; PPV: Positive predictive value;
PRISMA: Systematic Reviews and Meta-Analyses; PROSPERO: International prospective register of systematic reviews

\begin{abstract}
Acknowledgements
We would like to thank the librarian Christina Lindberg and statistician Hans Pettersson at Södersjukhuset, KISÖS for their help and contribution.
\end{abstract}

\section{Availability of data and materials}

All data generated or analyzed during this review can be found in the included articles.

\section{Authors' contributions}

KB and LK have contributed equally on each step of the study; design, screening of articles, analyzing the data and drafted the manuscript. Both authors approved the final manuscript.

Ethics approval and consent to participate

Not applicable.

\section{Consent for publication}

Not applicable.

\section{Competing interests}

KB has a position as consultant at SOS Alarm Sverige AB. There are no other competing interests and sources of funding.

\section{Publisher's Note}

Springer Nature remains neutral with regard to jurisdictional claims in published maps and institutional affiliations.

\section{Author details}

${ }^{1}$ Department of Clinical Science and Education, Södersjukhuset, Karolinska Institutet, SE 11883 Stockholm, Sweden. ²Department of Emergency Medicine, Södersjukhuset, Stockholm, Sweden. ${ }^{3}$ Department of Medical Sciences, Örebro University, Örebro, Sweden. ${ }^{4}$ Department of Emergency Medicine, Örebro University Hospital, Örebro, Sweden.

Received: 11 March 2018 Accepted: 5 July 2018

Published online: 09 November 2018

\section{References}

1. Snooks H, Evans A, Wells B, Peconi J, Thomas M, Woolard M, et al. What are the highest priorities for research in emergency prehospital care? Emerg Med J. 2009;26:549-50.

2. Medical Priority Dispatch system. http://www.emergencydispatch.org/Studies. Assessed 28 June 2018

3. Sporer KA, Johnson NJ, Yeh CC, Youngblood GM. Can emergency medical dispatch codes predict prehospital interventions for common 9-1-1 call types? Prehosp Emerg Care. 2008;12(4):470-8.

4. Andersen MS, Johnsen SP, Sörensen JN, Jepsen SB, Hansen JB, Christensen EF. Implementing a nationwide criteria-based emergency medical dispatch system: a register based follow-up study. Scand J Trauma Resusc Emerg Med. 2013:21:53.

5. Stipulante S, Tubes R, El Fassi M, Donneau AF, Van Troyen B, Hartstein G, et al. Implemention of the ALERT-algorithm, a new dispatcher-assisted cardio pulmonary resuscitation protocol, in non-advanced medical priority dispatch system (AMPDS) emergency medical services centres. Resuscitation. 2014;85:177-81.

6. Press W, Teukolsky S, Vetterling W, Flannery B. Modeling of data. Numerical recipes 3rd edition: the art of scientific computing, vol. 812. Cambridge, UK: Cambridge University Press; 2007

7. American College of Surgeons-COT. Resources for optimal care of injured patient. Chicago: American college of surgions-COT; 2006.

8. Moher D, Liberati A, Tetzlaff J, Altman DG, PRISMA Group. Preffered reporting items for systematic reviews and meta-analyses: the PRISMA statement. Br Med J. 2009;339:b2535.

9. Guyatt GH, Oxman AD, Visit GE, Kunz R, Falck-Ytter Y, Alonso-Coello P, et al. GRADE: an emerging consensus on rating quality of evidence and strength of recommendations. Br Med J. 2008;336:924-6. 
10. The GRADE approach for diagnostic tests and strategies. The GRADE Working Group 2000. http://gdt.guidelinedevelopment.org/app/handbook/ handbook.html\#h.f7lc8w9c3nh8. Assessed 28 June 2018.

11. Fleiss $J$, Cohen J, Everitt BS. Large sample standard errors of kappa and weighted kappa. Psychol Bull. 1969;72:323-7.

12. Landis JR. The measurement of observer agreement for categorical data. In: Koch GG, editor. Biometrics. Raleigh, North Carolina: The Biometric Society; 1977.

13. Dami F, Golay C, Pasquier M, Fuchs V, Carron PN, Hugli O. Prehospital triage accuracy in a criteria based dispatch Centre. BMC Emerg Med. 2015;15:32.

14. Moser A, Mettler A, Fuchs V, Hanhart W, Robert CF, Della Santa V, et al. Merger of two dispatch centres: does it improve quality and patient safety? Scand J Trauma Resusc Emerg Med. 2017;25(1):40.

15. Giannakopoulos GF, Bloemers FW, Lubbers WD, Christiaans HM, van Exter P, de Lange-de Klerk ES, et al. Criteria for cancelling helicopter emergency medical services (HEMS) dispatches. Emerg Med J. 2012;29(7):582-6.

16. Vaillancourt C, Charette M, Kasaboski A, Hoad M, Larocque V, Crete D, et al. Cardiac arrest diagnostic accuracy of 9-1-1 dispatchers: a prospective multicenter study. Resuscitation. 2015;90:116-20.

17. Deakin CD, England S, Diffey D. Ambulance telephone triage using 'NHS Pathways' to identify adult cardiac arrest. Heart. 2017;103(10):761-5.

18. Moller TP, Andrell C, Viereck S, Todorova L, Friberg H, Lippert FK. Recognition of out-of-hospital cardiac arrest by medical dispatchers in emergency medical dispatch centres in two countries. Resuscitation. 2016;109:1-8.

19. Fukushima $H$, Imanishi M, Iwami T, Kitaoka H, Asai H, Seki T, et al. Implementation of a dispatch-instruction protocol for cardiopulmonary resuscitation according to various abnormal breathing patterns: a population-based study. Scand J Trauma Resusc Emerg Med. 2015;23:64.

20. Tanaka Y, Nishi T, Takase K, Yoshita Y, Wato Y, Taniguchi J, et al. Survey of a protocol to increase appropriate implementation of dispatcher-assisted cardiopulmonary resuscitation for out-of-hospital cardiac arrest. Circulation. 2014;129(17):1751-60.

21. Gellerstedt M, Rawshani N, Herlitz J, Bang A, Gelang C, Andersson JO, et al. Could prioritisation by emergency medicine dispatchers be improved by using computer-based decision support? A cohort of patients with chest pain. Int J Cardiol. 2016;220:734-8.

22. Dami F, Emeryb A, Pasquierb M, Carronb P-N, Fuchsa V, et al. Proposition and operational characteristics of a new dispatch scale to specifically identify acute strokes. Eur J Emerg Med. 2017;24:202-7.

23. Viereck S, Moller TP, Iversen HK, Christensen H, Lippert F. Medical dispatchers recognise substantial amount of acute stroke during emergency calls. Scand J Trauma Resusc Emerg Med. 2016;24:89.

24. Malekzadeh J, Shafaee H, Behnam H, Mirhaghi A. The effect of Cincinnati prehospital stroke scale on telephone triage of stroke patients: evidencebased practice in emergency medical services. Int J Evid Based Healthc. 2015;13(2):87-92.

25. Clawson JJ, Scott G, Gardett I, Youngquist S, Taillac P, Fivaz C, et al. Predictive ability of an emergency medical dispatch stroke diagnostic tool in identifying hospital-confirmed strokes. J Stroke Cerebrovasc Dis. 2016; 25(8):2031-42

26. Krebes S, Ebinger M, Baumann AM, Kellner PA, Rozanski M, Doepp F, et al. Development and validation of a dispatcher identification algorithm for stroke emergencies. Stroke. 2012;43(3):776-81.

27. Wilmer I, Chalk G, Davies GE, Weaver AE, Lockey DJ. Air ambulance tasking: mechanism of injury, telephone interrogation or ambulance crew assessment? Emerg Med J. 2015:32(10):813-6.

28. Ball SJ, Williams TA, Smith K, Cameron P, Fatovich D, O'Halloran KL, et al. Association between ambulance dispatch priority and patient condition. Emerg Med Australas. 2016;28(6):716-24.

29. Ek B, Edstrom P, Toutin A, Svedlund M. Reliability of a Swedish pre-hospital dispatch system in prioritizing patients. Int Emerg Nurs. 2013;21(2):143-9.

30. Leopardi M, Sommacampagna M. Emergency nursing staff dispatch: sensitivity and specificity in detecting prehospital need for physician interventions during ambulance transport in Rovigo emergency ambulance service, Italy. Prehosp Disaster Med. 2013;28(5):523-8.

31. Riou M, Ball S, Williams TA, Whiteside A, et al. The linguistic and interactional factors impacting recognition and dispatch in emergency calls for out-of-hospital cardiac arrest: a mixed-method linguistic analysis study protocol. BMJ Open. 2017;7:e016510.

32. Buck BH, Starkman S, Eckstein M, et al. Dispatcher recognition of stroke using the National Academy Medical Priority Dispatch System. Stroke. 2009; 40:2027-30.
33. Chenaitia $\mathrm{H}$, Lefevre $\mathrm{O}, \mathrm{Ho} \mathrm{V}$, et al. Emergency medical service in the stroke chain of survival. Eur J Emerg Med. 2013;20:39-44.

34. Thomas SH, Biddinger PD. Helicopter trauma transport: an overview of recent outcomes and triage literature. Curr Opin Anaesthesiol. 2003;16: $153 e 8$.

35. Kurola J, Wangel M, Uusaro A, et al. Paramedic helicopter emergency service in rural Finland do benefits justify the cost? Acta Anaesthesiol Scand 2002;46:779e84

36. Oppe S, De Charro FT. The effect of medical care by a helicopter trauma team on the probability of survival and the quality of life of hospitalised victims. Accid Anal Prev. 2001:33:129e38.

37. Frankema SP, Ringburg AN, Steyerberg EW, et al. Beneficial effect of helicopter emergency medical services on survival of severely injured patients. Br J Surg. 2004;91:1520e6.

38. Mann NC, Schmidt TA, Cone DC. Defining research criteria to characterize medical necessity in emergency medical services: a consensus among experts at the Neely Conference. Prehospital Emerg Care Off J Natl Assoc EMS Physicians Natl Assoc State EMS Dir. 2004;8(2):138-53.

39. Castrén M, Karlsten R, Lippert F, Christensen EF, Bovim E, et al. Recommended guidelines for reporting on emergency medical dispatch when conducting research in emergency medicine: the Utstein style. Emergency Medical Dispatch expert group at the Utstein Consensus Symposium 2005. Resuscitation. 2008;79(2):193-7.

40. Fevang E, Lockey D, Thompson J, Lossius HM. Torpo Research Collaboration. The top five research priorities in physician-provided pre-hospital critical care: a consensus report from a European research collaboration. Scand J Trauma Resusc Emerg Med. 201;19:57

41. STARD 2015: An Updated List of Essential Items for Reporting Diagnostic Accuracy Studies. http://www.equator-network.org/reporting-guidelines/ stard/. Assessed 28 June 2018

\section{Ready to submit your research? Choose BMC and benefit from:}

- fast, convenient online submission

- thorough peer review by experienced researchers in your field

- rapid publication on acceptance

- support for research data, including large and complex data types

- gold Open Access which fosters wider collaboration and increased citations

- maximum visibility for your research: over $100 \mathrm{M}$ website views per year

At BMC, research is always in progress.

Learn more biomedcentral.com/submissions 